\title{
Comparative Study on the diversity Performance between Different Microstrip Antenna Arrays
}

\author{
Alaa H. Radhi, R. Nilavalan, H. S. Al-Raweshidy, and Nur Ab Aziz \\ College of Engineering, Design \& Physical Science, Brunel University, London, UK. \\ Email: Alaa.Radhi@brunel.ac.uk
}

\begin{abstract}
In this paper, a unique systematic comparative study between different microstrip antennas array types is presented. The study includes a comparison based on the mutual coupling $\left(S_{21}\right)$ and the envelope correlation coefficient between four different antennas array types: (a) PIFA antenna, (b) patch antenna, (c) monopole antenna, (d) slot antenna. All these different microstrip antennas have been designed and printed on a thin 1.6 mm FR-4 substrate with dielectric constant $\left(\varepsilon_{r}=4.4\right)$, suitable for wireless PCS applications (1.9 GHz). Numerical simulations are carried out using HFSS ver 17.0 software. The mutual coupling and envelope correlation coefficient between these different microstrip antennas are studied for various antenna separations. Also, another brief comparison is presented which based on the obtained results particularly in inputs return and radiation characteristics loss at fixed antenna separation, by determining the primarily associated parameters such as the bandwidth, gain, directivity, radiation efficiency. However; it is found that separation between multiple antenna elements is the most critical and crucial parameter affecting on the antenna diversity performance such as both the mutual coupling and the envelope correlation coefficient (primarily it will decrease linearly with the distance increment). This comparative study would be very helpful for RF design engineers to select suitable microstrip antenna type while designing antenna arrays at frequency 1.9 GHz for wireless PCS applications.
\end{abstract}

Index Terms - Microstrip antennas, Mutual coupling, PCS applications, Multiple-Input-Multiple-Output (MIMO).

\section{INTRODUCTION}

In MIMO systems, multiple antenna elements are required at both receiver and transmitter side [1]. The design of two or more antennas on a small mobile terminal for the MIMO systems is more challenging compared to the design of a conventional single antenna [2]. However; installing multiple antenna elements in the small space available in portable devices will inevitably cause severe mutual coupling and significantly degrades the diversity performance. Thus one of the main challenges to employing MIMO systems in mobile devices is the design of small MIMO antennas with lowest mutual coupling [2].A separation between multiple antenna elements is the most critical parameter affecting mutual coupling. Analytical studies have shown that for minimal or no mutual coupling, the distance between common antenna elements needs to be at least half wavelength [3].

With the rapid expansion of wireless MIMO communication systems, low profile, wide bandwidth, high isolation between antennas that maintain good performance is still in high demand. [3]. When a multiple-element antenna embeds into the small mobile terminal, it should be compact (low profile) as much as possible. Additional requirements should be met, e.g., low mutual coupling (a high isolation between antennas) and robustness while maintaining on the compactness with acceptable diversity performance for multiple antennas [4].Therefore, in designing the antenna for the mobile terminal, it is important to balance the trade-off between compactness and performance [1],[4]. In the literature; very few investigations have been done as a comparative study based on the diversity performance (such as mutual coupling and envelope correction coefficient) between more than two microstrip antennas types. However; this study presents a new comparative study between four different microstrip antenna array types (PIFA, patch, monopole, slot), having various dimensions with regular shapes such as rectangular and square. One of the important considerations in designing these antennas is maintaining on the compactness size and at the same time; to be working in a single narrow frequency band. However; in this work; all these antennas are proposed and designed to operate at frequency $1.9 \mathrm{GHz}$ band which is suitable for PCS applications. In this paper, diversity performances such as mutual coupling and envelope correlation coefficient for different antennas array types have been carried out to investigate the effect of the antenna separation. It provides a complete view compared with other works in the literature that most of them are focused only on just one or two common microstrip antenna types such as the patch or monopoles antennas only [5-6].All design and simulation, as well as optimization processes of these antennas, were done using High-Frequency Structure Simulation (HFSS) software version 17.0 from ANSYS that utilize to design and analyse the performance of different microstrip antennas in $3 \mathrm{D}$ environmental architecture.

\section{ANTENNA DESIGN AND CONFIGURATION}

The four different microstrip antennas are etched on an infinite thin $\mathrm{FR}_{4}$ substrate $\left(\varepsilon_{\mathrm{r}}=4.4\right.$ and $\left.h=1.6 \mathrm{~mm}\right)$ with a small infinite ground plane to be suitable for most mobile PCB circuit boards. A good impedance matching $\left(\mathrm{S}_{11}<-10 \mathrm{~dB}\right.$, VSWR $<2$ ) is achievable across the operating frequency $(1.9 \mathrm{GHz})$ for all the antenna designs. These antennas are separated by a distance $d$, which is made to vary from $0.3 \lambda_{0}$ to $1.0 \lambda_{0}$ to investigate an antenna diversity performance such as the mutual coupling and envelope correlation coefficient. Antenna feeding was either with $50 \Omega$ microstrip line that directly connected to the antenna or an appropriate coaxial pin. The impedance matching here can be obtained without the need for any additional matching element, by just selecting the proper feed position. The major advantage of this technique is 
its simplicity regarding fabrication and analysis, as well as impedance matching.

\section{A. Dual Microstrip PIFA Antenna Array}

The designed and geometrical parameters of the dual microstrip PIFAs antenna array are shown in Fig. 1. The PIFA antenna elements are also working at centre frequency $f_{c}=1.9$ $\mathrm{GHz}$ are placed collinearly along the $\mathrm{x}$-axis, and the minimum space distance of these antennas is $d=46 \mathrm{~mm}$ (corresponds 0.3 $\lambda_{0}$ approximately) measured from element centre to centre. Each PIFA element has a square patch outline with both equal width and length of $W=L=35 \mathrm{~mm}$ (corresponds $\lambda_{0} / 4$ approximately). The dimensions of the common ground plane and the substrate here are: $L_{g} \times W_{g}=90 \mathrm{~mm} \times 45 \mathrm{~mm}$, the radiating patch is made up of a very thin copper sheet with a thickness of $0.1 \mathrm{~mm}$.

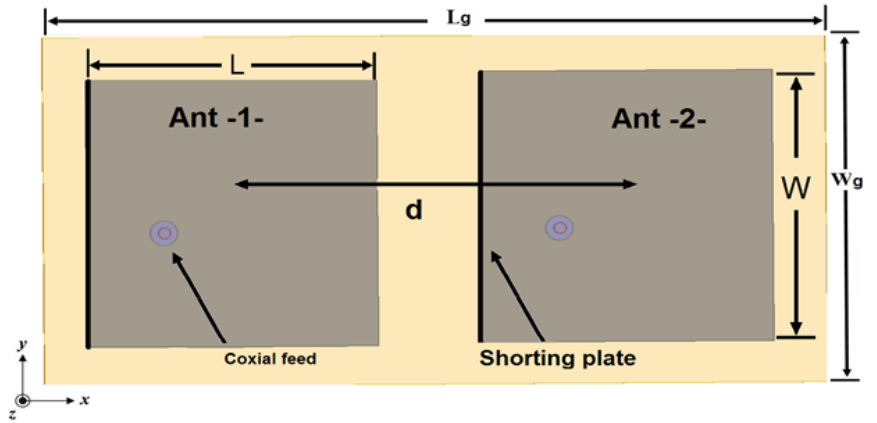

Fig. 1. Configuration of the dual PIFA antenna array (Top view).

Fig. 2. shows the return loss $\left(\mathrm{S}_{11}\right)$ and the mutual coupling $\left(\mathrm{S}_{21}\right)$ of dual PIFA antennas. The result of the return loss indicates that the PIFA antenna at $-10 \mathrm{~dB}$ has low $\mathrm{BW}$ of $0.036 \mathrm{GHz}$ (ranging from 1.9087 to $1.9447 \mathrm{GHz}$ ). The figure clearly shows that the strongest mutual coupling is found at the resonant frequency of the dual antenna elements, while the mutual coupling is decaying away from the PIFA resonance as the space between antennas linearly increases. An average of $44 \mathrm{~dB}$ reduction in the mutual coupling level is obtained as the distance is increased from $0.3 \lambda_{0}$ to $1.0 \lambda_{0}$.

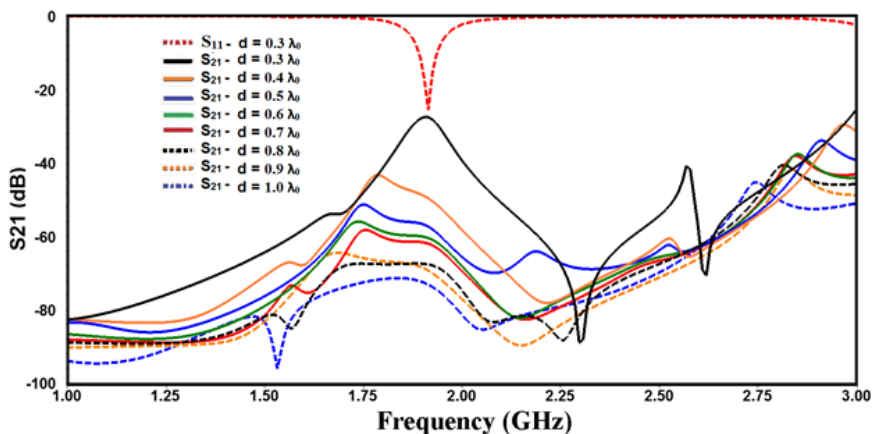

Fig. 2. The simulated mutual coupling of the PIFA antenna array when (d varying in terms of $\lambda 0$ ).

The simulated radiation patterns at $1.9 \mathrm{GHz}$ are illustrated in Figs. 3(a) and (b) on the realized gain on the z-x plane and zy plane; respectively. The radiation patterns are stable across the band; the maximums gain and directivity at $1.9 \mathrm{GHz}$ are, respectively, 2.7 and $3.8 \mathrm{dBi}$, with radiation efficiency of $70 \%$.

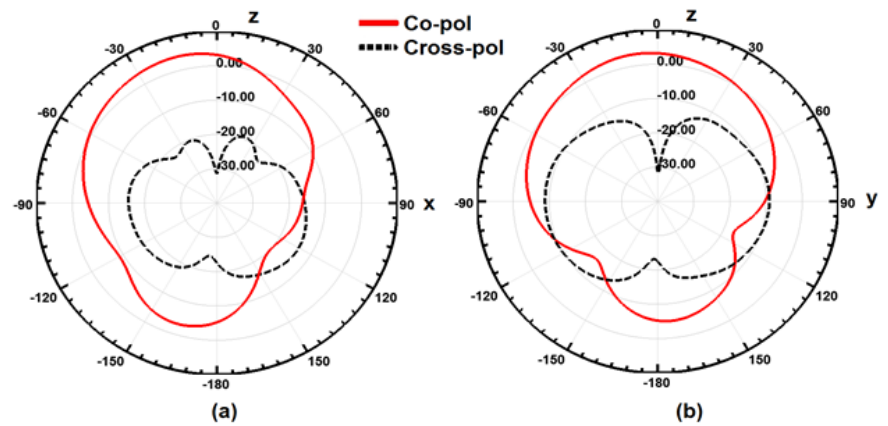

Fig. 3. Simulated radiation characteristics for dual PIFA antenna array at 1.9 GHz (a) Z-X plane,(b) Z-Y plane.

\section{B. Dual Microstrip Patch Antenna Array}

The designed and geometrical parameters of the dual microstrip patch antenna array are shown in Fig. 4. The antenna elements working at centre frequency $f_{c}=1.9 \mathrm{GHz}$ are placed collinearly along the $\mathrm{x}$-axis, the minimum space of the antennas is $d=48 \mathrm{~mm}$ (corresponds $0.3 \lambda_{0}$ approximately at the resonant frequency) measured from element centre to centre. Each patch element has a rectangular outline with width $W=$ $36 \mathrm{~mm}$ and length $L=45 \mathrm{~mm}$ (corresponds $0.28 \lambda_{0}$ approximately). The dimensions of the common ground plane and the substrate here are: $L_{g} \times W_{g}=100 \mathrm{~mm} \times 60 \mathrm{~mm}$.

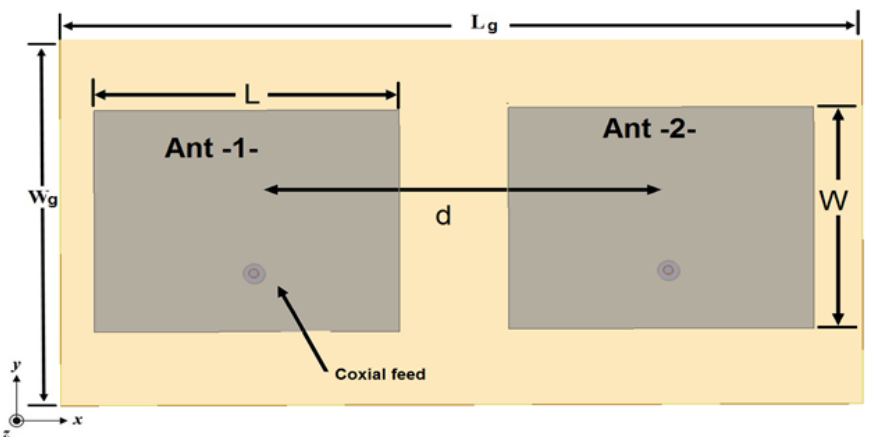

Fig. 4. Configuration of the dual patch antenna array (Top view).

Fig. 5. shows the return loss $\left(\mathrm{S}_{11}\right)$ and the mutual coupling $\left(\mathrm{S}_{21}\right)$ of dual patch antennas. The result of return loss shows that the patch antenna at $-10 \mathrm{~dB}$ has reasonable $\mathrm{BW}$ of 0.05 $\mathrm{GHz}$ (ranging from 1.914 to $1.964 \mathrm{GHz}$ ). The figure clearly shows that mutual coupling is decreasing linearly as the space between antennas increase. An average of $6 \mathrm{~dB}$ reduction in the mutual coupling level is obtained as the distance is increased from $0.3 \lambda_{0}$ to $1.0 \lambda_{0}$.

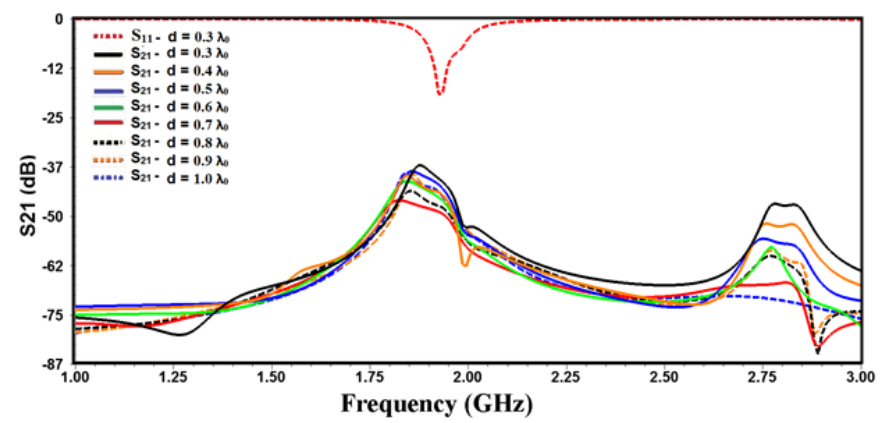

Fig. 5. The simulated mutual coupling of the patch antenna array when (d varying in terms of $\lambda_{0}$ ). 


\section{Brunel University, Draft Copy}

The simulated radiation patterns at $1.9 \mathrm{GHz}$ are illustrated in Figs. 6(a )and (b) on the realized gain on the z-x plane and z-y plane; respectively. The radiation patterns are stable across the band; the maximums gain and directivity at $1.9 \mathrm{GHz}$ are, respectively, 3.1 and $5.45 \mathrm{dBi}$, with radiation efficiency of $55 \%$.

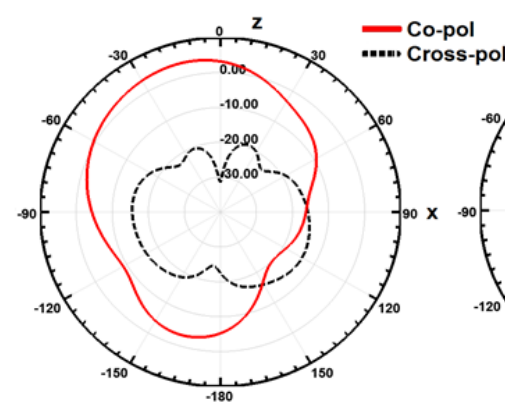

(a)

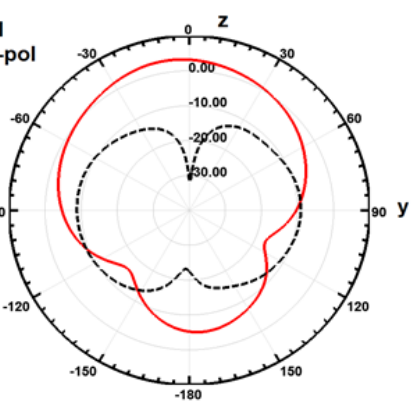

(b)
Fig. 6. Simulated radiation characteristics for dual patch antenna array at 1.9 GHz (a) Z-X plane,(b) Z-Y plane.

\section{Dual Microstrip Monopole Antenna Array}

The designed and geometrical parameters of the dual microstrip monopole antenna array are shown in Fig. 7. The monopole antenna elements working at centre frequency $f_{c}=$ $1.9 \mathrm{GHz}$ are placed collinearly along the $\mathrm{x}$-axis, the minimum space of these antennas is $d=45 \mathrm{~mm}$ (corresponds $0.3 \lambda_{0}$ approximately) and measured from element centre to centre. Each monopole element has a rectangular outline with width $W$ $=33 \mathrm{~mm}$ and length $L=47 \mathrm{~mm}$ (corresponds $0.28 \lambda_{0}$ approximately). The dimensions of the common substrate here are: $L_{s} \times W_{s}=105 \mathrm{~mm} \times 58 \mathrm{~mm}$.

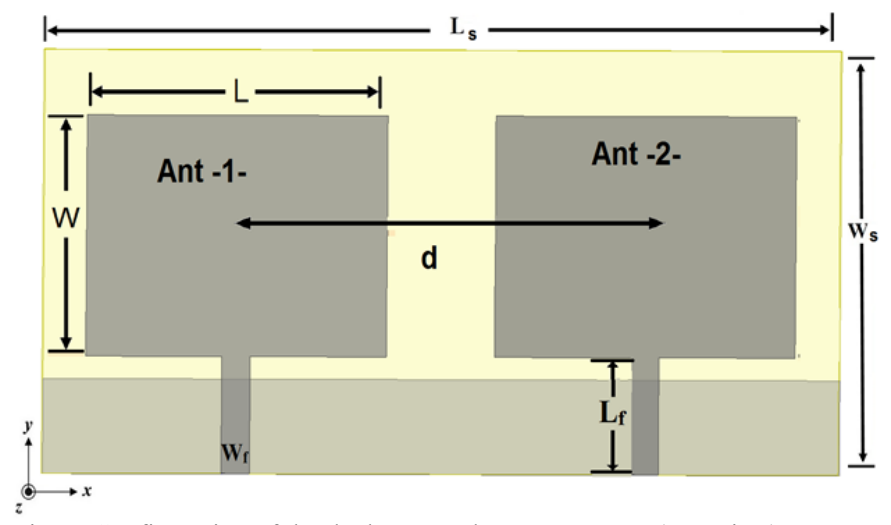

Fig. 7. Configuration of the dual monopole antenna array (Top view)

Fig. 8. shows the return loss $\left(\mathrm{S}_{11}\right)$ and the mutual coupling $\left(\mathrm{S}_{21}\right)$ of dual monopole antennas. The result of return loss indicates that the monopole antenna at $-10 \mathrm{~dB}$ has wide $\mathrm{BW}$ of $0.924 \mathrm{GHz}$ (ranging from 1.588 to $2.512 \mathrm{GHz}$ ). The figure clearly shows that the mutual coupling is decreasing linearly as the space between antennas increase. An average of $17 \mathrm{~dB}$ reduction in the mutual coupling level is obtained as the distance is increased from $0.3 \lambda_{0}$ to $1.0 \lambda_{0}$.

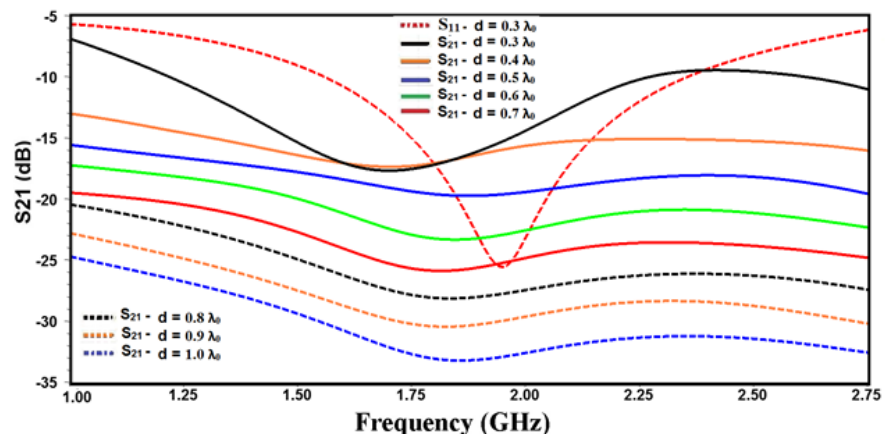

Fig. 8. The simulated mutual coupling of the monopole antenna array when (d varying in terms of $\lambda_{0}$ ).

The simulated radiation patterns at $1.9 \mathrm{GHz}$ are illustrated in Figs. 9(a) and (b) with respect to the realized gain on the $\mathrm{z}-\mathrm{X}$ plane and z-y plane; respectively. The radiation patterns are nearly Omni-directional across the band; the maximums gain and directivity at $1.9 \mathrm{GHz}$ are, respectively, 1.4 and $1.5 \mathrm{dBi}$, with radiation efficiency of $90 \%$.

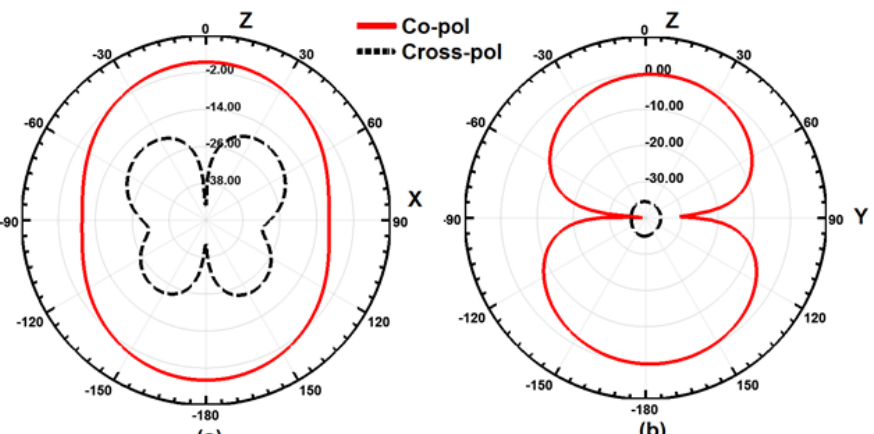

Fig. 9. Simulated radiation characteristics for dual monopole antenna array at $1.9 \mathrm{GHz}$ (a) Z-X plane,(b) Z-Y plane.

\section{Dual Microstrip Slot Antenna Array}

The designed and geometrical parameters of the dual microstrip slot antenna array are shown in Fig. 10. The slot antenna elements working at centre frequency $f_{c}=1.9 \mathrm{GHz}$ are placed collinearly along the $\mathrm{x}$-axis, and the minimum space of these antennas is $d=50 \mathrm{~mm}$ (corresponds $0.3 \lambda_{0}$ approximately) measured from element centre to centre. Each slot element has a rectangular outline plate with width $W=60$ $\mathrm{mm}$ and length $L=86 \mathrm{~mm}$ (corresponds $0.5 \lambda_{0}$ approximately). The dimensions of the common substrate here are: $L_{s} \times W_{s}=$ $114 \mathrm{~mm} \times 86 \mathrm{~mm}$.

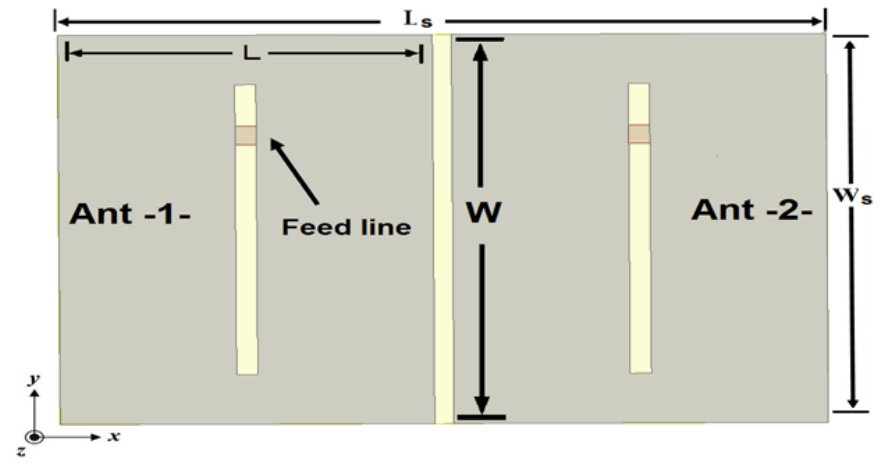

Fig. 10. Configuration of the dual slot antenna array (Top view) 
Fig. 11. shows the return loss (S11) and the mutual coupling (S21) of the dual monopole antennas. The result of return loss indicates that the monopole antenna at $-10 \mathrm{~dB}$ has moderate $\mathrm{BW}$ of $0.2959 \mathrm{GHz}$ (ranging from 1.7616 to 2.0575 $\mathrm{GHz}$ ). The figure clearly shows that the mutual coupling is decreasing linearly as the space between antennas increase. An average of $16 \mathrm{~dB}$ reduction in the mutual coupling level is obtained as the distance is increased from $0.3 \lambda 0$ to $1.0 \lambda 0$.

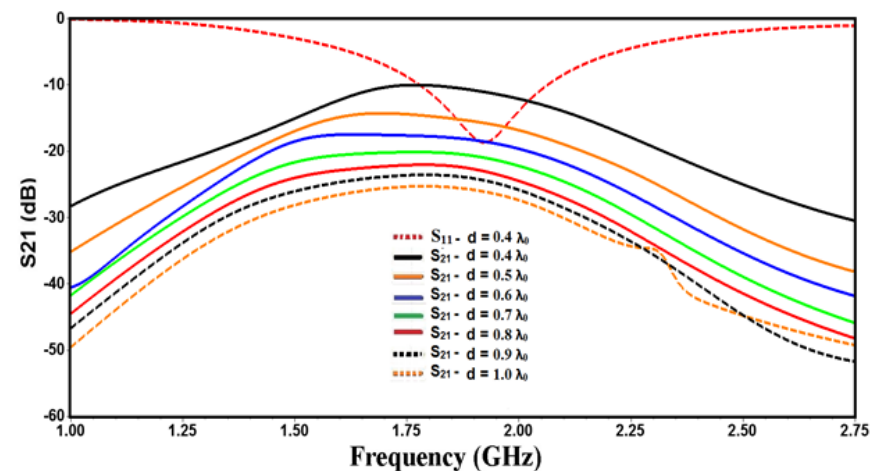

Fig. 11. The simulated mutual coupling of the slot antenna array when (d varying in terms of $\lambda_{0}$ ).

The simulated radiation patterns at $1.9 \mathrm{GHz}$ are illustrated in Figs. 12(a) and (b) on the realized gain on the z-X plane and $\mathrm{z}-\mathrm{y}$; respectively. The radiation patterns are nearly Omnidirectional across the band; the maximums gain and directivity at $1.9 \mathrm{GHz}$ are, respectively, 2.3 and $2.48 \mathrm{dBi}$, with radiation efficiency of $92 \%$.

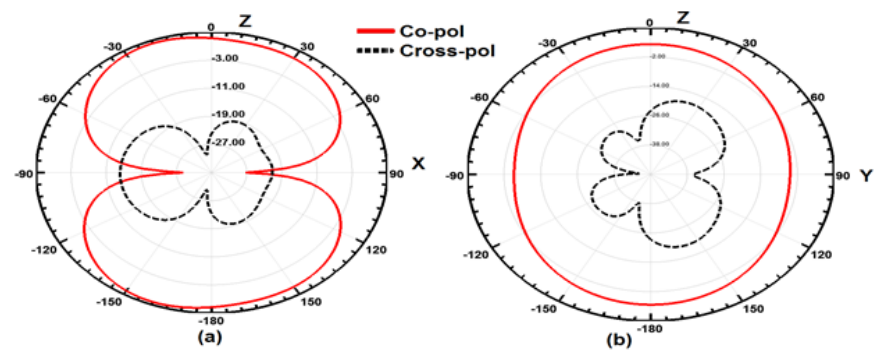

Fig. 12. Simulated radiation characteristics for dual slot antenna array at 1.9 GHz (a) Z-X plane, (b) Z-Y plane.

\section{PERFORMANCE SUMMARY AND COMPARISON}

\section{A. Mutual Coupling of Different Antennas Types at Varying Separation}

First; the mutual coupling of various antenna array types is investigated when the separation between these antennas are varying from $0.3 \lambda_{0}$ to $1.0 \lambda_{0}$ as presented in Table I

\begin{tabular}{|c|c|c|c|c|}
\hline Space & PIFA & Patch & Monopole & Slot \\
\hline $0.3 \kappa$ & -28 & -38.8 & -16.3 & -10 \\
\hline $0.4 \Lambda$ & -49 & -43.1 & -16.4 & -11 \\
\hline $0.5 \Lambda$ & -57.3 & -40.17 & -19.4 & -15.5 \\
\hline $0.6 \kappa$ & -60 & -43.9 & -23 & -18 \\
\hline $0.7 \kappa$ & -61.1 & -48.15 & -25.5 & -20.7 \\
\hline $0.8 \kappa$ & -67.6 & -46 & -27.7 & -22.5 \\
\hline $0.9 \kappa$ & -68.73 & -43.3 & -30 & -24 \\
\hline $1.0 \kappa$ & -72.6 & -44 & -33 & -26 \\
\hline
\end{tabular}

Table I Comparison table of the mutual coupling $\left(\mathrm{S}_{21}\right)$ in $\mathrm{dB}$ at $1.9 \mathrm{GHz}$ between four different antenna types at varying separation
In Fig. 13, the mutual coupling for the various antenna types is investigated when the separation between these antennas are varying from $0.3 \lambda_{0}$ to $1.0 \lambda_{0}$. From the simulated results, it clear that highest isolation (better case) can be obtained between two PIFA antennas of $\leq-40 \mathrm{~dB}$ (at separation $\geq 0.4 \lambda_{0}$ ), and smaller than the mutual coupling of the patch antennas, followed by monopole antennas and finally slot antennas suffering highest mutual coupling (worst case) compared with other antenna types.

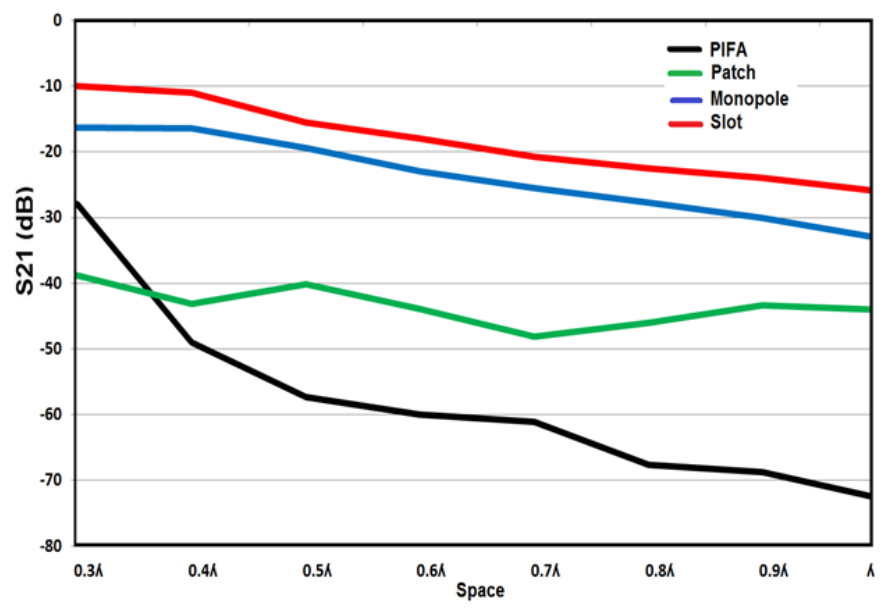

Fig. 13. Comparison of mutual coupling with different antennas types at varying separation (in terms of $\lambda_{0}$ ).

\section{B. Correlation of Different Antennas Types at Varying Separation}

Secondly; Envelope Correlation Coefficient (ECC) between antenna array elements is investigated according to space variation, Correlation coefficient one of the most important parameters to evaluate diversity performance because it is directly related to the antenna scattering parameters and may significantly degrade MIMO system performance. For dual antenna array elements, the envelope correlation coefficient equation using the scattering parameters is given by [7].

$$
\rho_{12}=\frac{\left|S^{*}{ }_{11} S_{21}-S^{*}{ }_{12} S_{22}\right|^{2}}{\left(1-\left|S_{11}\right|^{2}-\left|S_{21}\right|^{2}\right)\left(1-\left|S_{22}\right|^{2}-\left|S_{12}\right|^{2}\right)}
$$

In this work, ECC of different microstrip antenna array types was calculated according to (1) as presented in Table II based on assumptions: antenna system is lossless, and the antennas are excited separately, keeping the other antennas matched terminated.

\begin{tabular}{|c|c|c|c|c|}
\hline Space & PIFA & Patch & Monopole & Slot \\
\hline $0.3 \Lambda$ & -45 & -30 & -14.83 & -10.6 \\
\hline $0.4 \Lambda$ & -33 & -33.8 & -21.87 & -19.2 \\
\hline $0.5 \Lambda$ & -41 & -56 & -22 & -27.8 \\
\hline $0.6 \Lambda$ & -52.5 & -34 & -16 & -29.7 \\
\hline $0.7 \kappa$ & -53.9 & -33.16 & -22 & -33.5 \\
\hline $0.8 \Lambda$ & -51.8 & -36 & -24.4 & -38.8 \\
\hline $0.9 \Lambda$ & -58 & -41 & -23.1 & -40.2 \\
\hline $1.0 \Lambda$ & -70 & -45 & -17.35 & -42.1 \\
\hline
\end{tabular}

Table II Comparison table of the ECC in $\mathrm{dB}$ at $1.9 \mathrm{GHz}$ between four different antenna types at varying separation 
In Fig. 14, the envelope correlation coefficient for the different antenna types is investigated when the separation between these antennas are varying from $0.3 \lambda 0$ to $1.0 \lambda 0$. From the simulated results, the envelope correlation coefficient in the working band of the Patch antenna and PIFA antenna are respectively; lower than $-30 \mathrm{~dB}$ (at separation $\leq 0.5 \lambda 0$ ) and -40 $\mathrm{dB}$ (at separation $\geq 0.5 \lambda 0$ ), which are smaller than that other antenna types (slot and monopole). This observation indicates better behavior and diversity performance of MIMO antenna system will be achieved by using these antenna types (PIFA and patch).

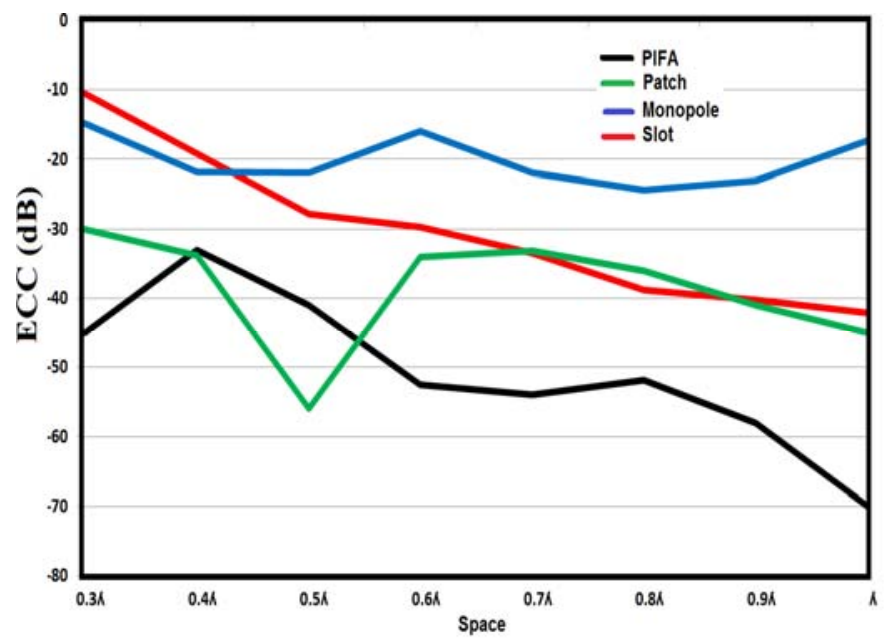

Fig. 14. Comparison of envelope correlation coefficient (ECC) with different antennas types at varying separation (in terms of $\lambda_{0}$ ).

\section{Performance of Different Antennas Types at Fixed Separation}

Finally, a comparison of various antenna performance parameters at resonant frequency including return loss, BW, gain, directivity and radiation efficiency for different microstrip antenna array types is shown in Table III at fixed minimum separation between these antennas $\left(d=0.3 \lambda_{0}\right)$.

\begin{tabular}{|c|c|c|c|c|c|c|}
\hline $\begin{array}{c}\text { Antenna } \\
\text { type }\end{array}$ & $\begin{array}{c}\mathbf{f}_{\mathbf{r}}, \\
\mathbf{G H z}\end{array}$ & $\begin{array}{c}\mathbf{S}_{\mathbf{1 1}} \mathbf{\text { dB }} \\
\mathbf{d B}\end{array}$ & $\begin{array}{c}\mathbf{B W} \\
\mathbf{\%}\end{array}$ & $\begin{array}{c}\text { Gain, } \\
\mathbf{d B i}\end{array}$ & $\begin{array}{c}\text { Dir, } \\
\mathbf{d B i}\end{array}$ & $\begin{array}{c}\text { Rad eff, } \\
\mathbf{\%}\end{array}$ \\
\hline PIFA & 1.93 & -23.2 & 1.90 & 2.7 & 3.8 & 70 \\
\hline Patch & 1.92 & -21 & 2.63 & 3.1 & 5.45 & 55 \\
\hline Monopole & 1.9 & -22 & 47 & 1.4 & 1.5 & 90 \\
\hline Slot & 1.9 & -20.8 & 15.58 & 2.3 & 2.48 & 92 \\
\hline
\end{tabular}

Table III Comparison table of the antennas performance between four different microstrip antenna types at fixed separation $\left(d=0.3 \lambda_{0}\right)$.

It is apparent from Table III that both PIFA and patch arrays achieve a narrower (lower) performance in terms of $\mathrm{BW}$, with a close values of 1.90 and $2.63 \%$, respectively, followed by the slot antenna array with a moderate BW value of $15.58 \%$ and finally the monopole antenna array achieves a wider BW performances with a value of $47 \%$. It should be noted that the considered BW corresponds to the frequency range over which VSWR is $<1.92$. Means a return loss of $10 \mathrm{~dB}$ or about $11 \%$ reflected power. As regards the radiation characteristics, the patch antenna array offers a higher directivity and gain with an average efficiency factor $(55 \%)$ as seen in the same table.In the second position comes the PIFA array that achieves a reasonable directivity of $3.8 \mathrm{~dB}$ with moderate radiation efficiency of $70 \%$, immediately followed by slot and monopole antenna arrays with highest radiation efficiency ( $\geq 90 \%$ ), with reasonable directivity of $2.48 \mathrm{~dB}$ and $1.5 \mathrm{~dB}$; respectively.

\section{CONCLUSION}

In this paper, four different microstrip antenna arrays (PIFA, patch, monopole, slot), with different dimensions and geometric shapes were proposed, characterised, and investigated for use at frequency $1.9 \mathrm{GHz}$ of wireless PCS application. All these antennas have been designed and printed on a thin $1.6 \mathrm{~mm}$ of none expensive $\mathrm{FR}_{4}$ substrate with dielectric constant $\left(\varepsilon_{\mathrm{r}}=4.4\right)$.A comprehensive comparative study between these various proposed microstrip antenna array types has been done, to understand the impact of the antenna separation on the diversity performances. It is found that the separation between multiple antenna elements is the most critical and crucial parameter affecting on both mutual coupling and envelope correlation coefficient. This comparison clearly proves that the PIFA array presents the best possible compromise in terms of performance, which has lower mutual coupling with very acceptable correlation coefficient and at the same time has a high directivity, reasonable gain and moderate radiation efficiency, However, its narrow BW, large dimensions compared with other antenna types at the same operating frequency constitute its major weak point compared with other antenna array types. This study would be helpful for RF engineers to select suitable microstrip antenna type while designing arrays for wireless PCS applications. As future work, practical implementation of these different microstrip antenna arrays types and simultaneous optimization using standard optimization procedures will be considered.

\section{REFERENCES}

[1] C. A. Balanis, Antenna Theory: Analysis and Design, 3rd Edition. Hoboken, NJ: John Wiley \& Sons, Inc., 2005.

[2] K. M. Prasanna and S. K. Behera, "Compact two-port UWB MIMO antenna system with high isolation using a fork-shaped structure," 2013 International Conference on Communication and Signal Processing, 2013.

[3] W. L. Stutzman and G. A. Thiele, Eds., Antenna Theory and Design,2nd ed. New York: Wiley, 1998.

[4] M. Salehi and A. Tavakoli, "A novel low mutual coupling microstrip antenna array design using defected ground structure," AEU - International Journal of Electronics and Communications, vol. 60, no. 10, pp. 718-723, 2006.

[5] R. Chair, A. Kishk, and K.-F. Lee, "Comparative study on the mutual coupling between different sized cylindrical dielectric resonators antennas and circular microstrip patch antennas," IEEE Transactions on Antennas and Propagation, vol. 53, no. 3, pp. 1011-1019, 2005.

[6] C. Hannachi and S. O. Tatu, "Performance comparison of $60 \mathrm{GHz}$ printed patch antennas with different geometrical shapes using miniature hybrid microwave integrated circuits technology," IET Microwaves, Antennas \& Propagation, vol. 11, no. 1, pp. 106-112, Aug. 2017.

[7] S. Blanch, J. Romeu, and I. Corbella, "Exact representation of antenna system diversity performance from input parameter description," Electron. Lett., vol. 39, no. 9, pp. 705-707, May 2003. 